\title{
Dosimetric measurements of Onyx embolization material for stereotactic radiosurgery
}

\author{
Donald A. Roberts ${ }^{\text {a) }}$ and James M. Balter \\ Radiation Physics Division, Department of Radiation Oncology, University of Michigan Hospital and Health \\ Systems, 1500 East Medical Center Drive, Ann Arbor, Michigan 48109 \\ Neeraj Chaudhary \\ Division of Neurointerventional Radiology, Departments of Radiology and Neurosurgery, University of \\ Michigan Hospital and Health Systems, 1500 East Medical Center Drive, Ann Arbor, Michigan 48109 \\ Joseph J. Gemmete \\ Division of Neurointerventional Radiology, Departments of Radiology, Neurosurgery, and Otolaryngology, \\ University of Michigan Hospital and Health Systems, 1500 East Medical Center Drive, \\ Ann Arbor, Michigan 48109
}

Aditya S. Pandey

Department of Neurosurgery, University of Michigan Hospital and Health Systems, 1500 East Medical Center Drive, Ann Arbor, Michigan 48109

(Received 16 April 2012; revised 18 September 2012; accepted for publication 18 September 2012; published 12 October 2012)

Purpose: Arteriovenous malformations are often treated with a combination of embolization and stereotactic radiosurgery. Concern has been expressed in the past regarding the dosimetric properties of materials used in embolization and the effects that the introduction of these materials into the brain may have on the quality of the radiosurgery plan. To quantify these effects, the authors have taken large volumes of Onyx 34 and Onyx 18 (ethylene-vinyl alcohol copolymer doped with tantalum) and measured the attenuation and interface effects of these embolization materials.

Methods: The manufacturer provided large cured volumes $(\sim 28 \mathrm{cc})$ of both Onyx materials. These samples were $8.5 \mathrm{~cm}$ in diameter with a nominal thickness of $5 \mathrm{~mm}$. The samples were placed on a block tray above a stack of solid water with an Attix chamber at a depth of $5 \mathrm{~cm}$ within the stack. The Attix chamber was used to measure the attenuation. These measurements were made for both 6 and $16 \mathrm{MV}$ beams. Placing the sample directly on the solid water stack and varying the thickness of solid water between the sample and the Attix chamber measured the interface effects. The computed tomography $(\mathrm{CT})$ numbers for bulk material were measured in a phantom using a wide bore CT scanner.

Results: The transmission through the Onyx materials relative to solid water was approximately $98 \%$ and $97 \%$ for 16 and $6 \mathrm{MV}$ beams, respectively. The interface effect shows an enhancement of approximately $2 \%$ and $1 \%$ downstream for 16 and $6 \mathrm{MV}$ beams. CT numbers of approximately 2600-3000 were measured for both materials, which corresponded to an apparent relative electron density (RED) $\rho_{e}^{w}$ to water of approximately 2.7-2.9 if calculated from the commissioning data of the CT scanner.

Conclusions: We performed direct measurements of attenuation and interface effects of Onyx 34 and Onyx 18 embolization materials with large samples. The introduction of embolization materials affects the dose distribution of a MV therapeutic beam, but should be of negligible consequence for effective thicknesses of less than $8 \mathrm{~mm}$. The measured interface effects are also small, particularly at $6 \mathrm{MV}$. Large areas of high-density artifacts and low-density artifacts can cause errors in dose calculations and need to be identified and resolved during planning. (C) 2012 American Association of Physicists in Medicine. [http://dx.doi.org/10.1118/1.4757918]

Key words: AVM, embolization, Onyx, stereotactic radiosurgery

\section{INTRODUCTION}

Cerebral arteriovenous malformations (AVMs) represent a cluster of abnormal arterioles, veins, and the interconnecting nidus without intervening cerebral tissue or capillary beds. Cerebral AVMs have a prevalence of 19 per 100000 patient years as reported by Brown et al. ${ }^{1}$ While AVMs can cause headaches, seizures, and ischemia, most commonly they present with intracranial hemorrhage. Cerebral AVM associated hemorrhage carries significant morbidity (40\%) and mortality $(12 \%){ }^{2}$ The goal of any treatment modality is to cause obliteration of the nidus thus preventing hemorrhage and associated complications.

Treatment of cerebral AVMs relies on a multimodality approach, which includes microsurgical resection, radiosurgery, and/or endovascular embolization. Endovascular techniques 
aim at preventing flow through a cerebral AVM by introducing embolization material within the nidus. While the flow may only diminish in most instances, smaller AVMs with few feeders can be cured with this technique. ${ }^{3}$ Two commonly used materials for cerebral AVM embolization are Onyx (ethylene-vinyl alcohol co-polymer $(\mathrm{EVOH})$ )and $n$-BCA ( $n$ butyl cyanoacrylate). ${ }^{4}$

Onyx is a commercial embolic agent consisting of a mixture of EVOH doped with micronized tantalum, which is suspended in a dimethyl sulfoxide (DMSO) carrier. The Onyx EVOH material is doped with tantalum $(z=73)$ to aid visualization during cerebral angiography. Onyx agents come in viscosities of 18,20 , and 34 centistokes $\left(\mathrm{mm}^{2} / \mathrm{sec}\right)$, the viscosities are achieved by changing the fraction of $\mathrm{EVOH}$ in the agent; the percentage of $\mathrm{EVOH}$ is $6 \%, 6.5 \%$, and $8 \%$, respectively, for the available viscosities. These forms of the Onyx material are referred to as Onyx 18, Onyx 20, and Onyx 34, respectively. Mamalui-Hunter et al. ${ }^{5}$ have concluded the concentration of tantalum in a sample of Onyx 34 they analyzed was $0.0784 \pm 0.012 \mathrm{~g} / \mathrm{cm}^{3}$.

Onyx is not "glue" as it has no actual adhesive properties. Instead solidification occurs when an Onyx agent contacts fluids, such as blood or other body fluids. The EVOH precipitates and the DMSO carrier flows out of the precipitated mass leaving the precipitated mass in place. The lower viscosity material is considered appropriate for use in larger malformations since it will penetrate more distally from injection, while the high viscosity material is appropriate for use to block areas of higher flow in larger vessels. Final solidification occurs within 5 min for Onyx agents.

A number of publications have reported lower obliteration rates and reduced success rates of patients treated with radiosurgery who have been embolized prior to treatment. ${ }^{6-9}$ Onyx may allow for a more complete occlusion of the AVM nidus based on its physical properties when compared with $n$-BCA. However, there are concerns that the tantalum visualization agent within the embolic material may have an effect on the efficacy of the treatment for those brain AVMs treated by radiosurgery. Tantalum causes significant artifacts on computed tomography (CT) exams and may make targeting of the lesion with radiosurgery difficult. In addition, tantalum has an atomic number $(Z=73)$, which highly attenuates the radiation at diagnostic energies. Other embolization materials such as $n$-BCA use iodine $(Z=53)$ as a visualization material during injection. The conjecture has been that the high $Z$ imaging material, tantalum or iodine, may also cause high attenuation at therapeutic energies causing errors in dose calculations, which could lead to less than optimal radiosurgery plans. If true, these suboptimal plans could be less efficacious in damaging the angioarchitecture of the AVM. Concerns have been expressed in the past regarding the dosimetric properties of embolizing agents such as Onyx and the effects that the introduction of these agents into the brain may have on the quality of the radiosurgery plans on based on this argument. ${ }^{10,11}$ A previous group has published results showing that dose deposition is not significantly affected by the presence of the embolization material for Gamma Knife treatments, which use ${ }^{60}$ Co treatment beams. ${ }^{5,12}$ Other direct mea- surements of attenuation constants for this embolization material have been done with small volumes $(\sim 1.5 \mathrm{cc})$ and with pinpoint chambers ${ }^{13}$ or in-phantom measurements, ${ }^{14}$ which may lead to problematic results due to the small sample sizes involved.

\section{METHODS AND MATERIALS}

The presence of visualization material during CT scans produces both bright regions of high absorption (high CT numbers) and dark regions of low absorption (low CT numbers). These areas correspond to regions of apparent high electron density and apparent low electron density, respectively. The resulting artifacts, which may obscure the adjacent target areas, cause delineation of target structures and dose calculations to become highly problematic. Figure 1 shows a $1 \mathrm{~mm}$ slice of a CT from an AVM patient that was embolized using Onyx 18 material. This figure illustrates CT artifacts due to the high $Z$ visualization material both in the immediate region of the embolization and well removed from that region. Additionally the question arises as to whether the presence of areas with high absorption in a CT scan made at kilovolt energies implies high dose deposition and attenuation at therapeutic energies in those same areas.

We made measurements of effective linear attenuation of bulk material, interface effects at therapeutic energies, CT numbers at diagnostic energies and performed model calculations of typical artifacts. The effective linear attenuation was measured relative to solid water to determine whether the embolization material affects dose deposition downstream from

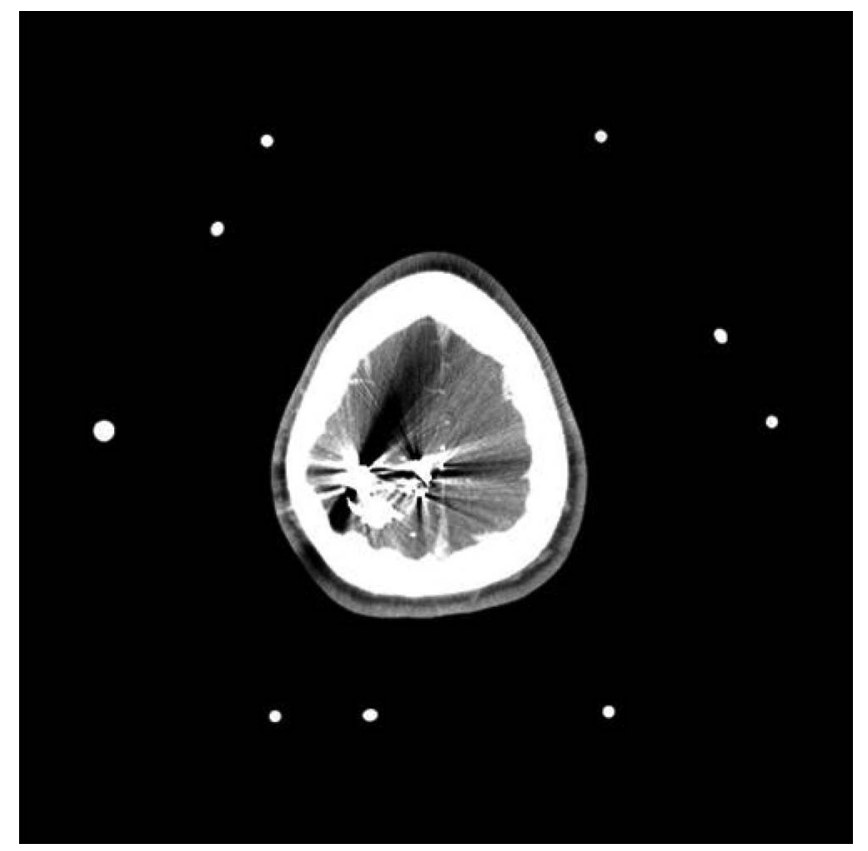

FIG. 1. The image above shows a $1 \mathrm{~mm}$ thick CT slice from a patient that had AVM embolization prior to SRS. Note the large artifacts caused by the tantalum powder infused into the embolization material to aid visualization. These artifacts could lead to large errors in dose calculations and obscure the target region making correct delineation of target volumes extremely problematic. 
the material. The interface effect was measured to determine if the presence of embolization material creates dose differences at the interface of the embolization material and the surrounding material. CT numbers were measured to establish the effect that the embolization material has on the apparent electron densities that are used in dose calculations both within the target region and in regions affected by artifacts created by the embolization material. Model calculations were made based on the measured CT numbers to illustrate dose errors created by incorrectly assigned apparent electron densities.

The properties of the embolization material were measured in bulk, since that represents a worst-case scenario. In actual use, the material forms an admixture of tissue and embolization material when it is injected. Neither the density nor exact composition of this admixture can be known, thus we have chosen to measure the effective linear attenuation of the bulk material, which then can be used as an upper limit for the linear attenuation of the in vivo admixture of tissue and embolization material.

To quantify these effects we have taken two large volumes of Onyx material and measured the attenuation and interface effects of these embolizing agents at linear accelerator (Linac) energies of 6 and 16 megavolts (MV). For this study we received from the manufacturer (EV3 Neurovascular, Irvine, California) a large volume $\left(\sim 28 \mathrm{~cm}^{3}\right)$ cured sample of Onyx 34 and a second cured sample of the same volume of Onyx 18. These cured samples of the materials were $8.5 \mathrm{~cm}$ in diameter with a nominal thickness of $5 \mathrm{~mm}$. The nominal thickness of the material was measured after the attenuation measurements were made. These large samples allowed direct measurements of both attenuation of MV treatment beams and interface effects using a Varian 2100 EX (Varian, Palo Alto, California) Linac at both 6 and $16 \mathrm{MV}$. For stereotactic radiosurgery (SRS) treatments only $6 \mathrm{MV}$ beams are used clinically in our institution. The additional measurements at $16 \mathrm{MV}$ were made for comparison of the attenuation properties of the material across typical energies available on clinical linear accelerators.

\section{II.A. Effective linear attenuation measurements}

For the attenuation measurements the samples were placed on a block tray in the accessory mount of the treatment Linac. A phantom was placed on the treatment couch below the accessory mount. The phantom consisted of a stack of solid water with a source to surface distance (SSD) of $95 \mathrm{~cm}$ with an Attix chamber at a depth of $5 \mathrm{~cm}$, as a result the top surface of the Attix chamber was at the machine's isocenter $(100 \mathrm{~cm})$. There was $11 \mathrm{~cm}$ of solid water below the Attix chamber to provide backscatter. The geometry for this measurement is shown in Fig. 2. The depth of $5 \mathrm{~cm}$ was chosen to avoid making measurements in buildup regions for both beams, which could lead to problematic interpretation of the results. For normalization, the sample was replaced with a $5 \mathrm{~mm}$ piece of solid water and the same measurements were repeated. By placing the samples on the block tray it was possible to use field sizes of up to $20 \times 20 \mathrm{~cm}^{2}$. Each measure-

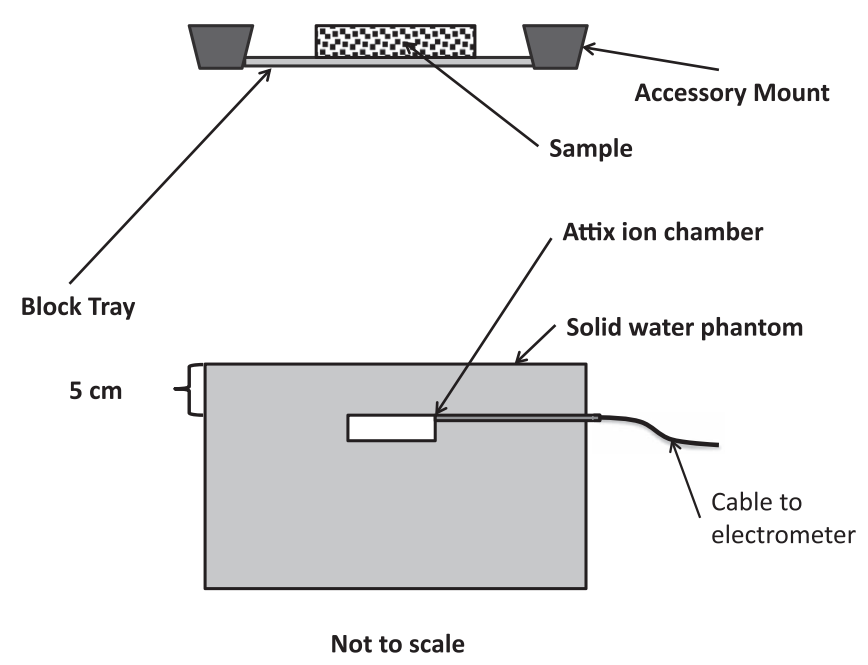

FIG. 2. The experimental setup used to measure the effective attenuation coefficients of the Onyx material. The sample material was placed on a block tray in the accessory head of the Linac. The phantom had a cross section of $40 \times 40 \mathrm{~cm}^{2}$ with a depth of $18 \mathrm{~cm}$ that provided $11 \mathrm{~cm}$ of backscatter below the Attix chamber.

ment was repeated five times. Estimated errors for these normalized transmission measurements were calculated from the standard deviation of dose measurements which were added in quadrature for the Onyx material and the solid water.

The effective attenuation constant was found by assuming that the transmission $(T)$ for material thickness $x$ can be written as

$$
T=e^{-\mu_{\text {eff } x}},
$$

where $\mu_{\text {eff }}$ is the effective attenuation constant. This effective attenuation constant was determined by inverting Eq. (1) to yield the following equation:

$$
\mu_{e f f}=\frac{-\ln (T)}{x} \text {. }
$$

In Eqs. (1) and (2), $T$ is the transmission of the material of thickness $x$. The transmission was measured for both the solid water and onyx embolization material by exposing the Attix chamber in the solid water stack, as described previously, to 200 monitor units with and without the material on the block tray. The transmission was calculated as the ratio of the dose measurements with the sample material on the block tray to the dose measurements with no material on the block tray. Measurements were made of the effective attenuation constant as a function of field size.

Errors in the effective attenuation constants were estimated by cutting each of the samples across the diameter of the sample in order to measure the sample thickness along the cut line with a pair of vernier calipers. A mean thickness and standard deviation of the material thickness were calculated from these data. The mean thickness was used as the value of $x$ in Eq. (2). The probable errors in the effective attenuation constants were estimated as errors associated with the measurement of $T$ and measurement of $x$ summed in quadrature:

$$
\sigma(\mu)=\sqrt{\left(\left(\frac{\sigma(T)}{x T}\right)^{2}+\left(\frac{\ln (T) \sigma(x)}{x^{2}}\right)^{2}\right)},
$$


where $\sigma(T)$ is the standard deviation of the measured normalized transmission as described previously and $\sigma(x)$ is the standard deviation of the thickness of the Onyx material determined from measurements.

To model the dose differences caused by inclusion of this embolization material in a therapy beam the following model was developed based on the measured effective attenuation constants. Estimates were made by taking the measured attenuation coefficients for Onyx 34 at $6 \mathrm{MV}$ and calculating the dose deposition difference at the distal edge of the embolizing material when that material was replaced by water while leaving the geometric distance, and hence the inverse square law, invariant. The calculations were water blocked. These errors are relative to water and not brain material and represent the error for a single beam which transits the embolizing material. The estimates of the probable errors were calculated by replacing the value of the attenuation coefficient with the mean value plus and minus one standard deviation. We note that this model accounts only for the loss of fluence due to the increased attenuation of the material and does not consider other factors such as change in scattering in the calculations, which would require extensive Monte Carlo calculations.

\section{II.B. Interface effects}

The interface effects were measured with an Attix chamber by placing the sample directly on the solid water stack and increasing the thickness of solid water between the sample and the Attix chamber by adding slabs of solid water one at a time while keeping the SSD to the top of the solid water stack constant at $95 \mathrm{~cm}$ and delivering 200 monitor units. The SSD of the stack was set with a front pointer after each addition of a solid water slab. The interface effect was measured for a $5 \times 5 \mathrm{~cm}^{2}$ field. A schematic drawing representing the setup used for the interface effect measurements is shown in Fig. 3. Replacing the embolization material with a $5 \mathrm{~mm}$ slab of solid water and repeating the measurement procedure normalized the measurements. The measurements were repeated five times for each depth of the embolization material and the

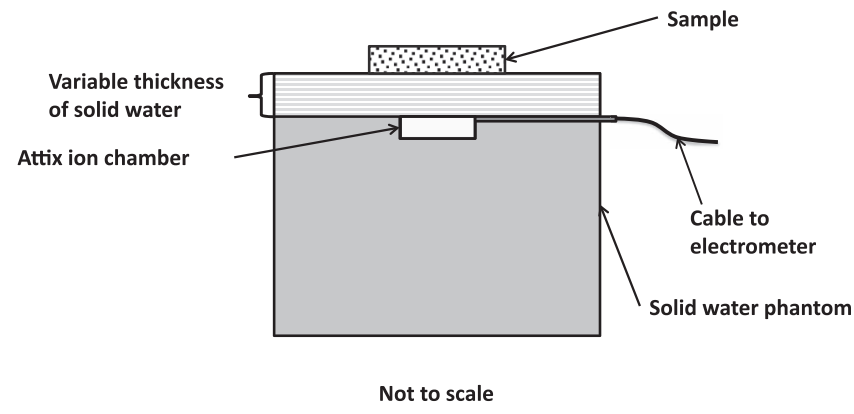

FIG. 3. Schematic representation of the experimental setup used to measure the interface effect of the Onyx embolization material. The Onyx sample material was placed directly on the top of the phantom and the thickness of the material between the Onyx sample and the Attix chamber was varied. The SSD was held constant for all measurements. The measurements were normalized to solid water by repeating the measurement with the Onyx sample replaced by a $5 \mathrm{~mm}$ thick piece of solid water. solid water. The ratio $R(x)$ (in percent) was then calculated as a function of the thickness $x$ of the solid water between the sample and the Attix chamber as

$$
R(x)=\left[\frac{D_{\text {Onyx }}(x)}{D_{\mathrm{SW}}(x)}\right] \times 100,
$$

where $D_{\text {Onyx }}(x)$ is the dose deposited at a depth of $x$ with the Onyx sample on top of the phantom and $D_{\mathrm{SW}}(x)$ is the dose deposited at a depth of $x$ with the $5 \mathrm{~mm}$ piece of solid water replacing the Onyx on top of the solid water phantom.

The interface effect at a depth of $x(\operatorname{IE}(x)$ in percent) from the Onyx sample was calculated as

$$
I E(x)=(R(x)-100) .
$$

The difference (in percent) calculated in Eq. (4) was interpreted as being caused by the replacement of the solid water with the embolization material at the interface, and thus due to the embolization material.

The errors for the interface effect were estimated by adding the standard deviations of the measurements of the dose for the Onyx material and solid water in quadrature.

\section{II.C. Measurements of CT numbers for embolization materials}

The radio-density of material measured in a CT scanner is measured in Hounsfield units, which are given by the following equation for material type denoted by $y$ :

$$
\operatorname{HU}(y)=1000 \frac{\left(\mu_{y}-\mu_{\text {water }}\right)}{\mu_{\text {water }}} .
$$

Equation (6) can be inverted to calculate the linear attenuation coefficient at the diagnostic energy of measurements of the material as follows:

$$
\mu_{y}=\frac{(\mathrm{HU}(y)+1)}{1000} \mu_{\text {water }},
$$

where $\mu_{y}$ and $\mu_{\text {water }}$ are the linear attenuation coefficients of material $y$ and water, respectively, in Eqs. (6) and (7). It is important to note, however, that the linear attenuation coefficient calculated in Eq. (7) is for the diagnostic energies used in the CT scan and not for the therapeutic energies used for treatment. Additionally, CT scanners do not accurately predict electron densities for high $Z$ material, but over respond ${ }^{15}$ creating artifacts. ${ }^{16-21}$

CT numbers were measured using a Philips Brilliance (Koninklijke Philips Electronics, Amsterdam, The Netherlands) large bore $85 \mathrm{~cm} 16$ slice CT scanner. The scanner CT numbers were calibrated to electron density using a Gammex 467 CT phantom (Gammex Inc. Middleton, WI) for CT numbers from -790 (120 kVp, LN-300 Lung phantom material) up to 1565 (120 kVp, cortical bone phantom material). The relative electron density (RED) was calculated by extrapolation from the clinical CT number to electron density curve used for treatment planning, which was determined during the commissioning of the scanner.

For these measurements samples of embolization material were placed between two pieces of solid water $5 \mathrm{~cm}$ thick with a $30 \times 30 \mathrm{~cm}^{2}$ cross section to mimic an in vivo scan. This 
Sagittal and Axial views

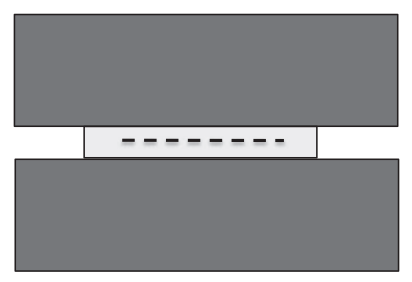

Solid water slabs

Onyx Sample

Region of interest
Coronal view

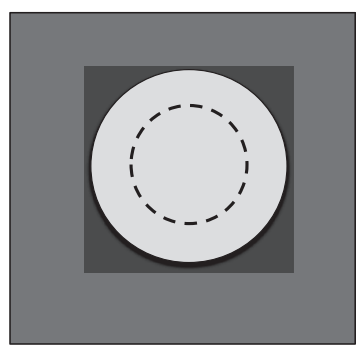

Not to scale

FIG. 4. Schematic representation of the placement of the region of interest (ROI) used to calculate the CT numbers of the Onyx samples. The sample was placed between two $30 \times 30 \mathrm{~cm}^{2}$ solid water slabs, shown above as dark material, the sample is shown as the lighter material. This phantom was scanned as described in the text. The region of interest was placed in the plane of the sample such that it was parallel to the surfaces of the sample and surfaces of the solid water slabs. The ROI was circular in the coronal plane as shown. The size of the ROI shown is exaggerated for clarity.

phantom was scanned with techniques of $90 \mathrm{kVp}, 120 \mathrm{kVp}$, and $140 \mathrm{kVp}$ with $1 \mathrm{~mm}$ thick CT slices. A circular region of interest (ROI) $1 \mathrm{~cm}$ in diameter was placed in the middle of the embolization material in the plane parallel to cross section of the material that appeared to be most centrally located relative to the thickness of the embolization material. Figure 4 illustrates the placement of the ROI used to measure the CT numbers in the samples. This assured that the region of interest used to calculate the CT numbers was not at the edge of the material and was therefore less likely to be affected by edge artifacts caused by the material. The software on the scanner was used to determine the mean values and the standard deviations of the CT numbers.

\section{II.D. Phantom calculations}

The presence of high $Z$ material, such as the Onyx embolization material, causes both high-density and low-density artifacts in CT scans. These artifacts are commonly seen with patients that have metal fillings in their teeth, hip or knee replacements, surgical clips, prostate seed implants, implantable cardiac devices and the leads to those devices to name just a few. ${ }^{16-21}$ A consequence of these artifacts is that the electron density calculated from the CT numbers within the regions of these artifacts in incorrect. This incorrect assignment of electron densities can significantly affect the quality of the dose calculations in these regions. In order to investigate the consequences of these artifacts on treatment plans of patients that have had embolization of their AVMs prior to obtaining the planning CT a simple mathematical phantom was constructed using the in house planning system, UMPlan at the University of Michigan.

Mathematical phantom used for model calculations

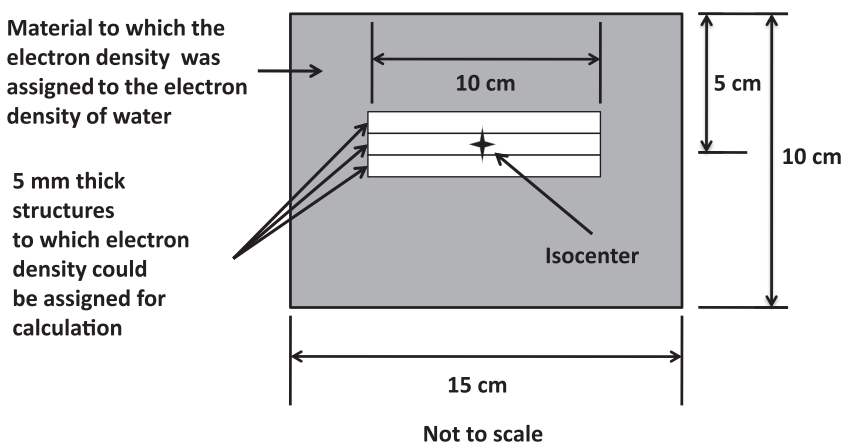

FIG. 5. Schematic representation of the mathematical phantom used for the model calculations. The main phantom was $10 \mathrm{~cm}$ thick with a cross section of $15 \times 15 \mathrm{~cm}^{2}$. The three structures indicated had cross sections of $10 \times 10 \mathrm{~cm}^{2}$ and thicknesses of $5 \mathrm{~mm}$. These structures could have their relative electron density assigned independently. The rest of the material had an electron density equal to water assigned. All the material had a mass density equal to water assigned. The drawing is not to scale, the variable structures have been drawn excessively thick for ease of viewing. All relevant dimensions are indicated on the drawing.

The model phantom was $10 \mathrm{~cm}$ thick with a cross section of $15 \times 15 \mathrm{~cm}^{2}$. The purpose of this phantom was to allow modeling of the effects of the artifacts created by the embolization agent on dose deposition calculations. Within the phantom three additional mathematical structures were defined each $5 \mathrm{~mm}$ thick with a cross section of $10 \times 10 \mathrm{~cm}^{2}$ such that the cross section for each structure was parallel to the cross section of the larger phantom, as shown in Fig. 5. Each of these structures formed a thin "slab" within the phantom. The phantom had a SSD of $95 \mathrm{~cm}$. The top of the first structure was $4.25 \mathrm{~cm}$ below the surface of the main phantom, $99.25 \mathrm{~cm}$ from the source. The two remaining structures had their top surfaces at 99.75 and $100.25 \mathrm{~cm}$, respectively. The center of the second slab was at isocenter, and the first and second slab could simulate material either proximal or distal to isocenter. The electron density of each slab structure could be assigned before calculation. The mass density was set to $1 \mathrm{~g} / \mathrm{cm}^{3}$ for these slab structures and the rest of the phantom. The portions of the phantom outside these three structures were assigned an electron density equal to water. This model is meant to demonstrate errors associated with artifacts caused by the embolization material independent of the errors caused by the material itself.

For these calculations a $3 \times 3 \mathrm{~cm}^{2} 6 \mathrm{MV}$ beam was defined such that the central ray passed through the phantom and each structure. The dose calculations used $1 \mathrm{~mm}$ grid spacing. Though there are many variations of electron density that can be calculated, five cases were chosen. The relative electron densities to water for the five model geometries chosen are given in Table II.

Geometry 1 is the baseline geometry where a $5 \mathrm{~mm}$ thick AVM is at isocenter with no artifacts surrounding it. The apparent electron density acquired from CT scans was assigned to the middle slab structure. The second geometry was meant to model a high-density artifact proximal to the AVM 
Onyx transmission relative to solid water at $5 \mathrm{~cm}$ depth

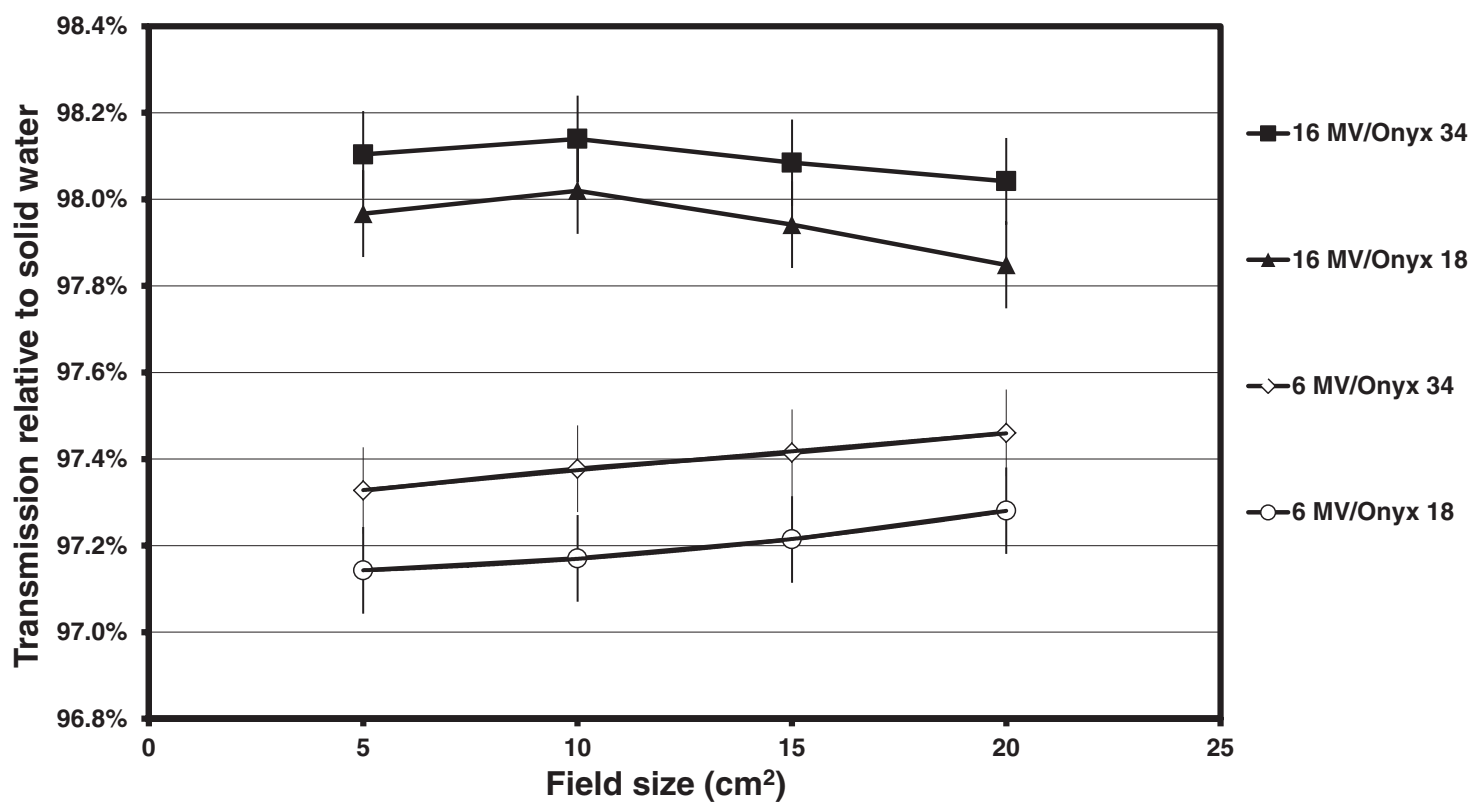

FIG. 6. The transmission relative to solid water for a $16 \mathrm{MV}$ beam (upper curves) and $6 \mathrm{MV}$ beam (lower curves) as a function of the field size. The beam energy (in MV) and material type are indicated in the legend. The error bars indicate one standard deviation and were calculated as indicated in the text.

followed by a low-density artifact distal. The third geometry was meant to approximate Onyx material with a distal high-density artifact followed by a low-density artifact. The fourth geometry had all structures assigned an electron density of water. This geometry was meant to simulate a waterblocked calculation, where the densities of any embolization material and associated artifacts were overridden. The last geometry was meant to approximate a case where the embolization material volume was correctly identified with the apparent electron density assigned from the CT scan followed by a low-density artifact downstream of the embolization material.

\section{RESULTS}

\section{III.A. Effective linear attenuation measurements}

The results of the measurements of the transmission of 6 and $16 \mathrm{MV}$ beams, normalized to $5 \mathrm{~mm}$ solid water, are shown in Fig. 6 for both Onyx 18 and Onyx 34. The relative transmissions were approximately $98 \%$ for $16 \mathrm{MV}$ and $97 \%$ for $6 \mathrm{MV}$ treatment beams. The error bars in Fig. 4 were calculated, as described in Sec. II.A, from the standard deviations of the measured doses for the Onyx material and solid water.

The effective linear attenuation coefficients calculated from Eq. (2) are shown in Fig. 7. The error bars in Fig. 7

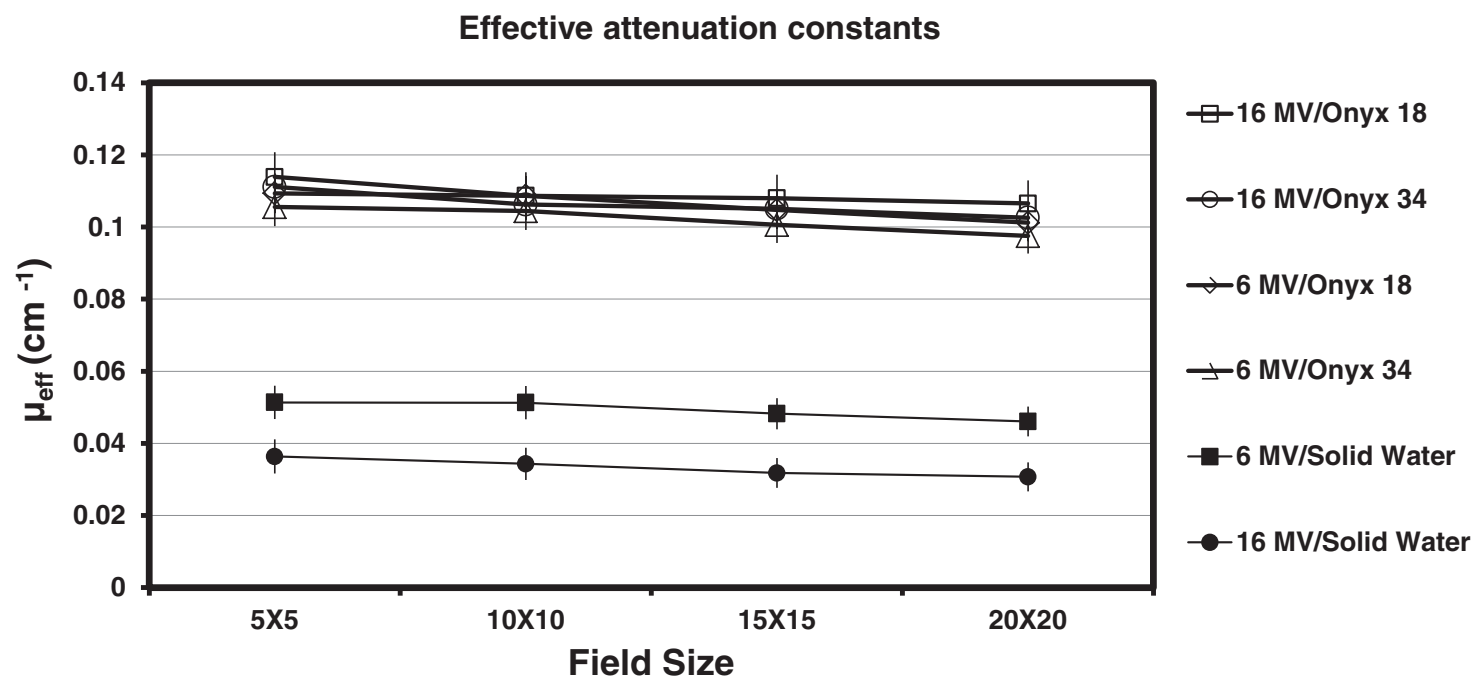

FIG. 7. The effective linear attenuation constants of solid water (bottom two curves) and the Onyx embolization materials at 6 and 16 MV. The data legend to the right of the figure indicates the energy of the beam (in MV) and the type of Onyx material. The error bars indicate one standard deviation and were calculated as indicated in the text. 
Probable error versus effective thickness of Onyx

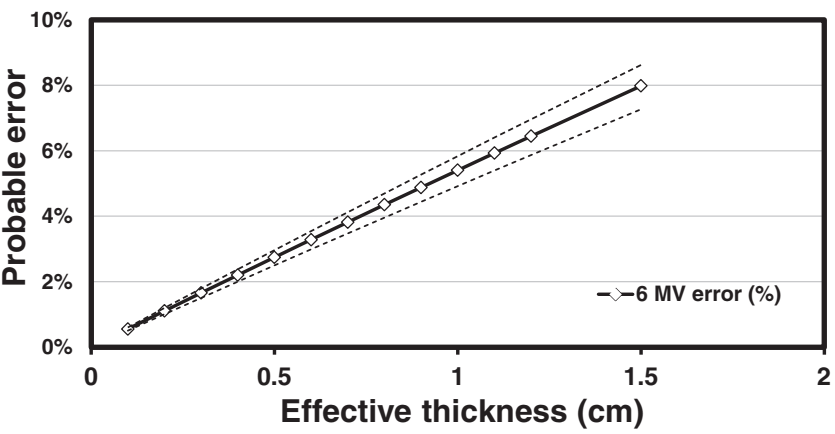

FIG. 8. The probable dosimetric error for a $6 \mathrm{MV}$ beam as a function of the effective thickness of the embolizing material relative to water for a single beam. The dashed lines represent the errors calculated by replacing the effective linear attenuation constant with the mean plus or minus one standard deviation.

were estimated from Eq. (3). Figure 7 indicates that effective attenuation constants for the two embolizing materials were 3-4 times higher than effective attenuation constants for solid water at both 6 and $16 \mathrm{MV}$. This indicates that this embolization material does introduce additional attenuation. At an effective thickness of $5 \mathrm{~mm}$ the attenuation of the Onyx material is approximately $2 \%$ higher for $16 \mathrm{MV}$ and $3 \%$ higher for $6 \mathrm{MV}$ than the attenuation of solid water.

The results of the model dose difference calculations are shown in Fig. 8. Probable errors are shown in Fig. 8 by dashed lines above and below the calculation line. There are two points to note from this figure: first, the model reproduces the transmission measurement shown in Fig. 4; secondly, the errors appear linear with thickness because the thicknesses shown in the figure are small compared to $1 / \mu_{\text {eff }}$ causing the exponential nature of the attenuation to appear linear in the displayed range.

\section{III.B. Interface effects}

Figure 9 shows the results of the interface measurements for Onyx 34; the results for Onyx 18 are similar. Figure 9(a) shows the results of the measurements of the interface effect on Onyx 34 for a $6 \mathrm{MV}$ beam. The vertical axis represents the excess, or deficit, when the sample is replaced with a $5 \mathrm{~mm}$ thick slab of solid water. The inset in Fig. 9(a) shows an expanded view for depths from 0 to $20 \mathrm{~mm}$ of the interface effect of a $6 \mathrm{MV}$ beam to better illustrate the effect at small distances. Figure 9(b) shows the results for a 16 MV beam. From these figures it is apparent that at $16 \mathrm{MV}$ there does appear to be a small interface effect on the order of $2 \%$ in the first $10 \mathrm{~mm}$. For $6 \mathrm{MV}$, the dose is apparently enhanced at the interface by about $1 \%$, but the magnitude of the interface effect is approximately equal to the measurement uncertainty. If any real positive interface effect exists at $6 \mathrm{MV}$ it is probably limited to not more than $5 \mathrm{~mm}$ and has a magnitude of less than $1 \%$. At $6 \mathrm{MV}$ an apparent deficit of approximately $0.5 \%$ in the region from 10 to $50 \mathrm{~mm}$ is seen. This deficit was reproducible over a number of experiments. No similar deficit was measured for the $16 \mathrm{MV}$ beam; the interface effect decreases

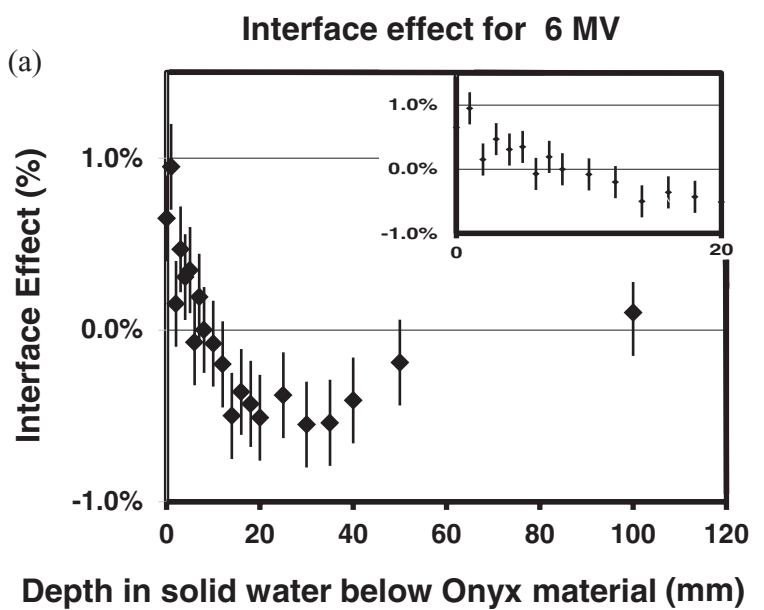

(b) Interface effect for $16 \mathrm{MV}$

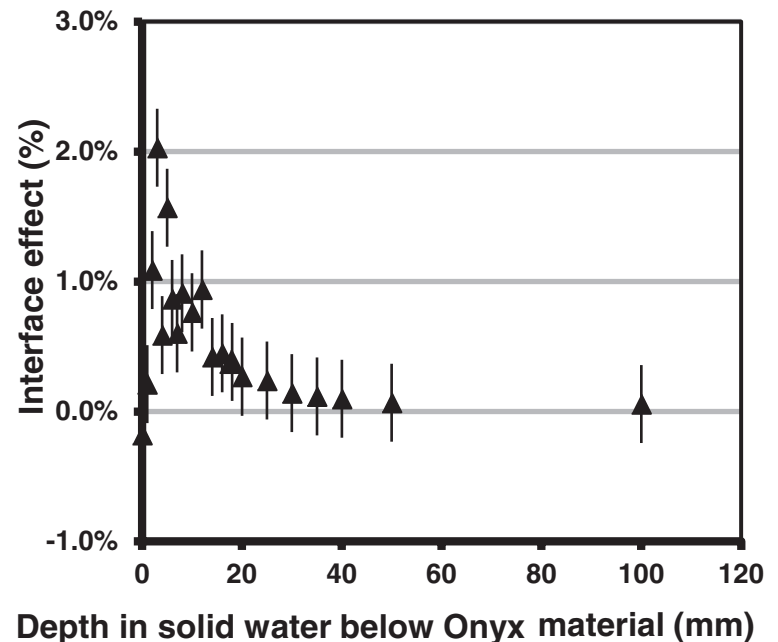

FIG. 9. Graph (a) shows the results of the measurements of the interface effect on Onyx embolization materials for $6 \mathrm{MV}$ beam. The inset in the corner shows an expanded view of the measurements for depths between $0 \mathrm{~mm}$ and $20 \mathrm{~mm}$ to better illustrate the interface effect for a $6 \mathrm{MV}$ beam. Graph (b) shows the results of measurements for a $16 \mathrm{MV}$ beam. The interface effect was calculated as the ratio of the dose deposited in an Attix chamber at the indicated depth below the Onyx material and below a $5 \mathrm{~mm}$ slab of solid water, respectively. A field size of $5 \times 5 \mathrm{~cm}^{2}$ was used for these measurements. For each measurement the SSD was kept constant. The error bars indicate one standard deviation and were calculated as indicated in the text.

to zero, within experimental uncertainty, at approximately $20 \mathrm{~mm}$ below the material for this higher beam energy.

\section{III.C. CT number measurements}

Table I shows the results of the measurements of CT numbers and the value $\rho_{e}^{w}$ for the electron density relative to water. As noted in Sec. II.C high $Z$ materials cause the CT scanners to over-respond. This over-response coupled with the inherent errors associated with the extrapolation of CT numbers to high HU results in values of $\rho_{e}^{w}$ for the embolizing agents which are un-physically high. The high values of the CT numbers measured in the CT scanner and the physical size of the samples created bright artifacts in the portions of the 
TABLE I. Measured CT numbers and calculated electron density relative to water $\left(\rho_{e}^{w}\right)$ for Onyx materials. The relative electron densities were calculated using the clinical CT number to electron density curve which was determined during the commissioning of the CT scanner and are excessively high.

\begin{tabular}{|c|c|c|c|c|}
\hline \multirow[b]{2}{*}{ Technique (kVp) } & \multicolumn{2}{|c|}{ Measured CT numbers } & \multicolumn{2}{|c|}{$\rho_{e}^{w}$} \\
\hline & Onyx 34 & Onyx 18 & Onyx 34 & Onyx 18 \\
\hline 90 & $2950 \pm 246$ & $2685 \pm 230$ & $2.86 \pm 0.239$ & $2.69 \pm 0.230$ \\
\hline 120 & $2975 \pm 268$ & $2615 \pm 221$ & $2.84 \pm 0.256$ & $2.65 \pm 0.224$ \\
\hline 140 & $2997 \pm 279$ & $2685 \pm 249$ & $2.89 \pm 0.269$ & $2.71 \pm 0.252$ \\
\hline
\end{tabular}

phantom slabs that were either directly above or below the sample. These bright artifacts essentially affected all portions of the phantom material on slices where the Onyx material was present.

\section{III.D. Phantom model calculations}

To compare the results of the five model phantom calculations described in Sec. II, the ratio of cases 2-5 was formed by dividing the computed dose on the central axis by the computed dose from geometry 1 . The results are seen in Fig. 10.

The first point to note from this figure is that the waterblocked calculation, geometry 4 , actually has the largest deviations from geometry 1 . Secondly, the major errors in the dose calculations do not necessarily occur near high-density structures, but rather propagate downstream several centimeters and become nearly constant at a distance of $\sim 3 \mathrm{~cm}$ downstream of these structures. Geometry 5 , which is closest to geometry 1 , has the smallest deviations. Geometries with large low-density regions tend to show excess dose downstream because the attenuation of the material has been underestimated. Likewise, the model with the largest high-density artifact, geometry 3 shows a deficit of dose downstream due to the overestimate of the attenuation caused by the apparent high-density region of the artifact. These calculations show quickly the consequences that both high- and low-density artifacts caused by the embolization material can have on dose calculations algorithms. Care must be exercised to assure that the dose calculations will not be adversely affected by the presence of artifacts in planning CT scans.

\section{DISCUSSION}

From Fig. 6 it is seen that for an effective embolizing material thickness of $5 \mathrm{~mm}$, the dose difference between solid water and Onyx material is $2 \%$ and $3 \%$ for 16 and $6 \mathrm{MV}$ beams, respectively. The embolization material does show a greater linear attenuation coefficient than solid water, as can be seen in Fig. 7 where $\mu_{\text {eff }}$ increases by about a factor of three for the embolization material relative to solid water at therapeutic energies. Due to the increase in the linear attenuation coefficient for the Onyx material the dosimetric differences can become significant $(>5 \%)$ for beams which encounter effective material thickness greater than $8-10 \mathrm{~mm}$ as seen in Fig. 8. Thus for beams passing through large amounts of embolizing material, greater than $8 \mathrm{~mm}$ of effective thickness, a correction to monitor units may be required if the target is distal to the Onyx material.

The treatment of AVMs using SRS is generally limited to malformations where the maximal size is usually less than

\section{Ratio of central axis dose to model geometry}

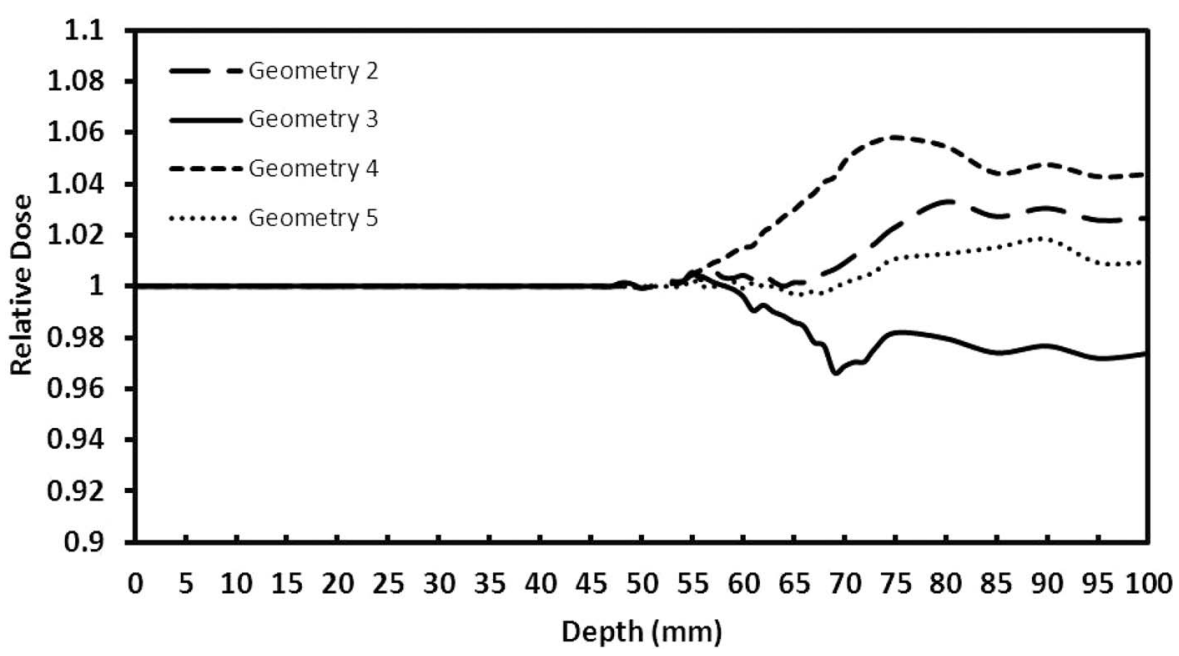

FIG. 10. The ratio of dose deposition for the four geometries divided by the baseline geometry as discussed in the text with the parameters given in Table II. The lines represent the errors, which may be caused by incorrect assignment of electron densities caused by CT artifacts in the planning scan. 
TABLE II. Electron densities relative to water $\left(\rho_{e}^{w}\right)$ used in phantom model structures for model calculations and simulation purposes as described in the text.

\begin{tabular}{|c|c|c|c|c|}
\hline \multirow[b]{2}{*}{ Geometry } & \multicolumn{3}{|c|}{$\begin{array}{c}\rho_{e}^{w} \\
\text { Phantom structure number }\end{array}$} & \multirow[b]{2}{*}{ Purpose of simulation } \\
\hline & 1 & 2 & 3 & \\
\hline 1 & 1 & 2.9 & 1 & AVM volume with electron density from assigned CT scan \\
\hline 2 & 2.9 & 0.001 & 0.001 & Low-density artifact distal to AVM \\
\hline 3 & 2.9 & 2.9 & 0.001 & High and low-density artifacts surrounding AVM \\
\hline 4 & 1 & 1 & 1 & Water blocked calculation \\
\hline 5 & 1 & 2.9 & 0.001 & Low density artifact distal to AVM \\
\hline
\end{tabular}

$3 \mathrm{~cm}$ in any dimension. ${ }^{22-24}$ However, the exact nature of the angioarchitecture of each AVM is unique, so it is not possible to estimate a priori the effective thickness of the embolizing material within an AVM. This is a clinical decision that will have to be discussed at the time of treatment, first to decide if monitor units corrections are needed, and secondly to make a clinical estimate of the effective thickness of the embolizing material seen by each treatment beam.

The measured interface effects shown in Fig. 9 are not significant $(<1 \%)$ for $6 \mathrm{MV}$; while a small enhancement of $\sim 2 \%$ or less is seen at $16 \mathrm{MV}$. This effect is limited to about $20 \mathrm{~mm}$ for the higher energy beam. The curve seen in Fig. 9(a) for the $6 \mathrm{MV}$ beam shows a tantalizing hint of interesting physics. There may be two competing processes in place, which may cause the observed behavior. First the presence of the high $Z$ tantalum will increase Compton scattering in the embolization material creating a small increase of dose close to the interface. This may be competing with the increased attenuation which removes photon fluence from the beam downstream, and thus is responsible for the apparent dose deficit just beyond the interface. In this model the higher energy $16 \mathrm{MV}$ beam should scatter even more electrons toward the interface, while the lower attenuation $(\sim 1 \%)$ of the $16 \mathrm{MV}$ beam could be just enough to account for the lack of dose deficit beyond $10 \mathrm{~mm}$ as seen in Fig. 9(b), as the higher energy beam is less attenuated as seen in Fig. 6. Confirmation of this theory would require significant Monte Carlo calculations, which are beyond the scope of this paper. However, this theory does seem to conform to the physics and qualitatively explain the results. We conclude however, that any interface effect at $6 \mathrm{MV}$ is small and can be ignored for treatment beams.

Figure 1 shows a $1 \mathrm{~mm}$ slice of a CT from an AVM patient that was embolized using Onyx 18 material. Note the high CT numbers in the area of the embolization as well as the areas of both high and low CT numbers due to artifacts caused by the embolization material. These artifacts arise from the high CT numbers of the Onyx material, as indicated in Table I. These artifacts are very likely to obscure the treatment volume, making accurate definition of treatment volume problematic at best. To establish target volumes for these patients it is recommended that other forms of imaging, such as digital subtraction angiography (DSA), magnetic resonance angiography (MRA), computed tomography angiography (CTA), or MRI, ${ }^{25,26}$ should be obtained according to the clinical practice of each institution. These other imaging modalities should be used to establish target volumes and then registered to the CT scans. Corrections to monitor units can be made if deemed clinically relevant based on the error estimates shown in Fig. 8 after image fusion and target transfer.

Several issues exist in mitigating dose calculation uncertainties arising from CT scans of patients with embolization material. First, care must be used if a water blocked calculation is considered, as assigning an electron density of water to the bulk material may well exacerbate the errors in the dose calculations as shown in Fig. 10 for geometry 4. Secondly, dose errors in calculations tend to propagate distally from the edge of the high-density structure. While these dosimetric errors may be large several centimeters from this edge they can be $<2 \%$ at or near the embolization material, which is considered to be near or in the target volume. In this case the actual volume of the embolization material can be reassigned a bulk density equal to the surrounding material. If that is not possible then Fig. 8 can be used to estimate the error in the dose calculations and monitor units can be recalculated as needed.

A recent publication ${ }^{5}$ has suggested that for Linac based radiosurgery low energy components of the therapy beams could be responsible for under-dosing of target areas in SRS cases based on Monte Carlo calculations of the peak and mean energy of a $6 \mathrm{MV}$ Linac. ${ }^{27}$ Neither the attenuation measurements nor the interface effect results, as described in this paper, support that hypothesis.

This group concluded that embolization material had a negligible effect on the delivered dose for ${ }^{60} \mathrm{Co}$ treatment from a Gamma Knife. While ${ }^{60} \mathrm{Co}$ is at a different energy (1.17 and $1.33 \mathrm{MeV}$ ) compared to our measurements (6 and $16 \mathrm{MV}$ ) the measurements made in this paper are in qualitative agreement with the previous results for ${ }^{60} \mathrm{C}$. Figure 8indicates that dosimetric errors greater than $5 \%$ will only occur for effective embolizing material thickness that are in excess of $8 \mathrm{~mm}$ for a $6 \mathrm{MV}$ beam.

\section{CONCLUSIONS}

Attenuation effects and interface effects of Onyx on the delivered dose to the target may be small for MV Linac treatment beams. However, if the effective thickness of the embolization material is greater than $8 \mathrm{~mm}$ modifications to monitor unit calculations may be necessary to properly reflect the increased attenuation of the Onyx material and assure correct target dose. 
A potentially large effect of the embolization material on patient outcome could be due to poor volume delineations caused by artifacts in, and around, the target volume if a CT scan is utilized. The high $Z$ doping of the embolization material, which is used for visualization during embolization, causes these artifacts. To establish target volumes for these patients it is recommended that other forms of imaging be obtained and fused to the planning CT. This allows the target volumes to be transferred to the $\mathrm{CT}$ scans after registration and thus avoids target delineation errors associated with high $Z$ CT artifacts. Volumes outside the target area that exhibit artifacts in the CT scan should have their electron densities over-ridden to electron densities appropriate to the tissue in those areas prior to planning.

\section{ACKNOWLEDGMENTS}

The authors gratefully acknowledge the help of Martha Matuszak in preparing the mathematical phantom used in the dose calculations. Dr. Pandey has received research grants from EV3, Microvention. The Onyx material used in this study was provided by EV3 as part of these research grants.

a) Author to whom correspondence should be addressed. Electronic mail: darobert@med.umich.edu

${ }^{1}$ R. D. Brown, D. O. Wiebers, J. C. Torner, and W. M. O'Fallon, "Incidence and prevalence of intracranial vascular malformations in Olmsted County, Minnesota, 1965 to 1992," Neurology 46, 949-952 (1996).

${ }^{2}$ J. van Beijnum, C. E. Lovelock, C. Cordonnier, P. M. Rothwell, C. J. Lijn, and R. A. Salman, "Outcome after spontaneous and arteriovenous malformation-related intracerebralhemorrhage: Population-based studies," Brain 132, 537-543 (2009).

${ }^{3}$ D. G. Abud, R. Riva, G. S. Nakiri, F. Padovani, M. Khawaldeh, and C. Mounayer, "Treatment of brain arteriovenous malformations by double arterial catheterization with simultaneous injection of Onyx: Retrospective series of 17 patients," Am. J. Neuroradiol. 32, 152-158 (2011).

${ }^{4}$ Y. Loh and G. R. Duckwiler, "A prospective, multicenter, randomized trial of the Onyx liquid embolic system and N-butyl cyanoacrylate embolization of cerebral arteriovenous malformations," J. Neurosurg. 113, 733-741 (2010).

${ }^{5}$ M. Mamalui-Hunter, T. Jiang, K. M. Rich, C. P. Derdeyn, and R. E. Drzymala, "Effect of liquid embolic agents on Gamma Knife surgery dosimetry for arteriovenous malformations," J. Neurosurg. 115, 364-370 (2011).

${ }^{6}$ B. E. Pollock, J. C. Flickinger, L. D. Lunsford, A. Maitz, and D. Kondzolka, "Factors associated with successful arteriovenous malformation radiosurgery," Neurosurgery 42, 1239-1247 (1998).

${ }^{7}$ B. E. Pollock, D. Kondzolka, L. D. Lunsford, D. Bissonette, and J. C. Flickinger, "Repeat stereotacticradiosurgery of arteriovenous malformation: Factors associated with incomplete obliteration," Neurosurgery 38, 318-324 (1996).

${ }^{8}$ L. Miyawaki, C. Dowd, W. Wara, B. Goldsmith, N. Albright, P. Gutin, V. Halbach, G. Hieshima, R. Higashida, B. Lulu, L. Pitts, M. Schell, V. Smith, K. Weaver, C. Wilson, and D. Larson, "Five year results of LINAC radiosurgery for arteriovenous malformations: Outcome for large AVMS,” Int. J. Radiat. Oncol. Biol. Phys. 44, 1089-1106 (1999).

${ }^{9}$ S. L. Blackburn, W. W. Ashley, Jr., K. M. Rich, J. F. Simpson, R. E. Drzymala, W. Z. Ray, C. J. Moran, D. T. Cross, M. R. Chicoine, R. G. Dacey, Jr., C. P. Derdeyn, and G. J. Zipfel, "Combined endovas- cular embolization and stereotactic radiosurgery in the treatment of large arteriovenous malformations," J. Neurosurg. 114, 1758-1767 (2011).

${ }^{10}$ Y. M. Andrade-Souza, M. Ramani, D. Scora, M. Tsao, K. ter Brugge, and M. Schwartz, "Embolization before radiosurgery reduces the obliteration rate of arteriovenous malformations," Neurosurgery 60, 443-452 (2007).

${ }^{11}$ Y. M. Andrade-Souza, M. Ramani, D. J. Beachey, D. Scora, M. N. Tsao, K. ter Brugge, and M. L. Schwartz, "Liquid embolization material reduces the delivered radiation dose: A physical experiment," Acta Neurochir. Suppl. (Wien) 50, 161-164 (2008).

${ }^{12}$ M. Mamalui-Hunter, T. Jiang, C. Derdyn, D. Low, and R. Drzymala, "The effect of embolization glue on Gamma Knife radiosurgery of arterio-venous malformations," Med. Phys. 36, 2794-2797 (2009).

${ }^{13}$ N. Shtrauss, S. Alani, S. Mimon, D. Schifter, and A. Kanner, "Onyx embolization effects on diagnostic images for radiosurgery in AVM patients," Med. Phys. 36, 2598-2599 (2009).

${ }^{14}$ F. Bing, R. Doucet, F. Lacroix, J. P. Bahary, T. Darsaut, D. Roy, F. Guilbert, J. Raymond, and A. Weill, "Liquid embolization material reduces the delivered radiation dose: Clinical myth or reality?” Am. J. Neuroradiol. 33, 320-322 (2012).

${ }^{15}$ C. Coolens, and P. J. Childs, "Calibration of CT Hounsfield units for radiotherapy treatment planning of patients with metallic hip prostheses: The use of extended CT-scale," Phys. Med. Biol. 48, 1591-1603 (2003).

${ }^{16}$ M. Kachelrieß, O. Watzke, and W. A. Kalender, "Generalized multidimensional adaptive filtering for conventional and spiral single-slice, multi-slice, and cone-beam CT," Med. Phys. 28, 475-490 (2001).

${ }^{17}$ J. Hsieh, Image Artifacts: Appearances, Causes and Corrections in Computed Tomography: Principle Design, Artifacts and Recent Advances (SPIE, Bellingham, WA, 2003), pp 167-240.

${ }^{18}$ P. M. Silverman, L. D. Spicer, R. McKinney, Jr., and D. B. Feldman, "Computed tomographic evaluation of surgical clip artifact: Tissue phantom and experimental animal assessment," Comput. Radiol. 10, 37-40 (1986).

${ }^{19}$ W. A. Kalender, R. Hebel, and J. Ebersberger, "Reduction of CT artifacts caused by metallic implants," Radiology 164, 576-577 (1987).

${ }^{20}$ Y. Zhang, L. Zhang, X. R. Zhu, A. K. Lee, M. Chambers, and L. Dong, "Reducing metal artifacts in cone-beam CT images by preprocessing projection data," Int. J. Radiat. Oncol. Biol. Phys. 67, 924-932 (2007).

${ }^{21}$ M. R. Ay, A. Mehranian, M. Abdoli, P. Ghafarian, and H. Zaidi, "Qualitative and quantitative assessment of metal artifacts arising from implantable cardiac pacing devices in oncological PET/CT studies: A phantom study," Mol. Imaging Biol. 13, 1077-1087 (2011).

${ }^{22}$ H. Kano, L. D. Lunsford, J. C. Flickinger, H. Yang, T. J. Flannery, N. R. Awan, A. Niranjan, J. Novotny, and D. Kondziolka, "Stereotactic radiosurgery for arteriovenous malformations, Part 1: Management of Spetzler-Martin Grade I and II arteriovenous malformations," J. Neurosurg. 116, 11-20 (2012).

${ }^{23}$ H. Kano, D. Kondziolka, J. C. Flickinger, H. Yang, T. J. Flannery, N. R. Awan, A. Niranjan, J. Novotny, and L. D. Lunsford, "Stereotactic radiosurgery for arteriovenous malformations, Part 3: Outcome predictors and risks after repeat radiosurgery," J. Neurosurg. 116, 21-32 (2012).

${ }^{24}$ H. Kano, D. Kondziolka, J. C. Flickinger, K. Park, P. V. Parry, H. Yang, S. Sirin, A. Niranjan, J. Novotny, and L. D. Lunsford, "Stereotactic radiosurgery for arteriovenous malformations, Part 6: Multistaged volumetric management of large arteriovenous malformations," J. Neurosurg. 116, 54-65 (2012).

${ }^{25}$ K. D. Hamm, J. Klisch, G. Surber, G. Kleinert, C. Eger, and R. Aschenbach, "Special aspects of diagnostic imaging for radiosurgery of arteriovenous malformations," Neurosurgery 62, A44-A52 (2008)

${ }^{26}$ M. Morikawa, Y. Numaguchi, D. Rigamonti, T. Kuroiwa, M. I. Rothman, G. H. Zoarski, J. M. Simard, H. Eisenberg, P. P. Amin, "Radiosurgery for cerebral arteriovenous malformations: Assessment of early phase magnetic resonance imaging and significance of gadolinium-DTPA enhancement," Int. J. Radiat. Oncol. Biol. Phys. 34,663-675 (1996).

${ }^{27}$ D. Sheikh-Bagheri, and D. W. Rogers, "Monte Carlo calculation of nine megavoltage photon beam spectra using BEAM code," Med. Phys. 29, 391-402 (2003). 